\title{
Journal's responsibility in maintaining scientific integrity
}

\author{
Li-Li Yang $^{1} \cdot$ Si-Yun Xu ${ }^{1} \cdot$ Zhi-Yi Yang $^{1} \cdot$ Zheng-Yan Zhao $^{1}$. Qiang Shu ${ }^{1}$
}

Received: 10 November 2021 / Accepted: 21 November 2021 / Published online: 4 January 2022

(c) Children's Hospital, Zhejiang University School of Medicine 2021

Scientific integrity has been regarded as a priority in scientific development in China [1]. The Chinese governmental agencies have paid unprecedented attentions on promoting scientific integrity in recent years. In May 2018, the Communist Party of China Central Committee and the State Council issued guidelines on promoting scientific integrity [2]. Ever-harsher punishments for researchers who committing scientific misconduct were introduced by the Chinese government [3]. In November, 2018, 43 penalties for major academic misconduct were endorsed by 41 national government agencies. Meanwhile, the Ministry of Science and Technology of China (MOST) were established a notification system for reporting cases involving in scientific misconduct and the related penalties publicly.

Since June 8, 2021, MOST has reported 103 cases involving scientific misconduct in medical sciences, which have been investigated and handled by medical institutions [4]. All these cases have been reported publicly on the websites of national institutions including China Research Integrity Website (https://www.orichina.cn). These cases are alarming for researchers to abide by academic credit line and scientific ethics. We found that all the 103 cases were involving misconducts in publications; the top three misconducts are falsification and fabrication of data, inappropriate authorship, and academic paper selling or ghostwriting. The cases revealed what a medical journal can do in maintaining medical research integrity.

We outline the following six strategies that World Journal of Pediatrics (WJP) has adopted to maintain scientific integrity (Table 1).

Zheng-Yan Zhao

Zhaozy@zju.edu.cn

Qiang Shu

shuqiang@zju.edu.cn

1 National Clinical Research Center for Child Health, National Children's Regional Medical Center, Children's Hospital, Zhejiang University School of Medicine, Hangzhou 310052, China

\section{Quality control}

WJP adopts the internal screening plus external peer-review system for paper quality control. As a journal co-published with Springer Nature, all the manuscripts submitted will be provided a crude screening by Springer Nature editorial team on ethics, plagiarism, and duplicate publication. Then, the manuscripts will be assigned to the Editor-in-Chief (EIC). The EIC will assign the manuscripts to the journal's medical editors for initial screening. Medical editors make internal editorial screening on all aspects including scope, formatting, declarations and ethics, plagiarism or duplicate submission, and data completion. Manuscripts will be sent out for peer-reviewing after meeting all the editorial screening criteria. One of the peer-reviewers is the journal's statistician who will evaluate the study design, statistical analysis and data interpretation independently. EIC will make the final decision for all manuscripts.

\section{Data availability}

The journal asks authors to provide a data availability statement for the submitted manuscripts. Currently, authors are encouraged to share data at public data reservoir and provide a linkage if possible. If the authors prefer not sharing the data in the data reservoir, they should provide the data if asked by the readers, reviewers or editors. Step-by-step, the journal will make data sharing mandatory. We now have asked the authors to provide raw data to the statistician for checking the results independently when necessary.

\section{Transparent conflict of interest declaration}

Conflict of interest (COI) is within the category of scientific integrity [5]. Transparent disclosure of COI is the most effective way to manage $\mathrm{COI}$ in medical research, because $\mathrm{COI}$ will affect how information is presented in scientific journals and in medical education forums [6]. Our previous study revealed 
Table 1 Six strategies for maintaining scientific integrity in World Journal of Pediatrics

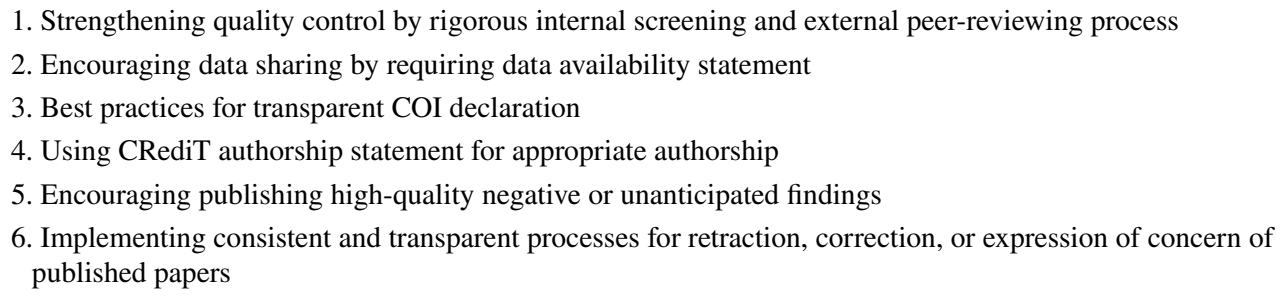

1. Strengthening quality control by rigorous internal screening and external peer-reviewing process

2. Encouraging data sharing by requiring data availability statement

3. Best practices for transparent COI declaration

4. Using CRediT authorship statement for appropriate authorship

6. Implementing consistent and transparent processes for retraction, correction, or expression of concern of

that failure to disclose COI may be related to journals' bad COI policy practice or researchers' inadequate knowledge and misunderstanding of COI disclose policy $[5,7]$. Therefore, the journal requires all authors to use updated ICMJE format to declare their COI upon submissions. The best COI policy for the journal needs to be studied and practiced further.

\section{CRediT authorship statement}

Inappropriate authorship is a threat to the integrity of scientific publication [8] and now is one of the top scientific misconducts in the cases reported publicly in China [4]. CRediT authorship statement can help in recognizing individual author contributions, reducing authorship disputes, and facilitating collaboration [9]. WJP requires authors to provide a CRediT authorship statement during the submission process. The section of "Author contributions" will appear in the published papers. Now, any disputes on authorship change should be approved by the EIC; furthermore, the journal will not permit the authorship changes during the revision stage.

\section{Negative or unanticipated findings}

Negative findings are equally important to be published to complete the available picture in areas of scientific inquiry [10]. Kretser et al. recommend using the "anticipated" and "unanticipated" to describe "positive" and "negative" results [11]. They encourage journals to publish "unanticipated" results. Not publishing the null or negative results may lead to a waste of human and financial resources which are dedicated to addressing the same, previously addressed research questions [11]. As a pediatric journal, WJP has always been encouraging authors to publish high-quality negative or unanticipated findings in pediatrics.

\section{Timely and transparent post-operation processes}

Authors and the journal have responsibilities to correct or retract the paper if an error or research misconduct was detected [12]. WJP has adopted consistent and transparent are processes for retraction, correction, or expression of concern of published papers. If errors is identified or suspicion of presence of misconduct is identified after publication, the journals and/or the publisher Springer Nature will carry out an investigation procedure following COPE guidelines [12]. Depending on the nature and severity of the errors, erratum, expression of concern or retraction will be implemented. Erratum will be published on a numbered page and will be linked to the original article. Editorial expression of concern or retraction notice will also be published and linked to the original article. For the papers requiring editorial expression of concern or retraction, the author's institution may be informed.

WJP is now transfering to a monthly publication from the 18 th issue in 2022. The journal will publish papers meeting standards of quality and scientific integrity via implementing rigorous review process, adhering to updated publication ethics, and handling concerns of scientific misconduct after publication timely and transparently.

Author contributions YLL: writing —original draft. XSY, YZY, ZZY, and SQ: writing-review and editing.

Funding This study was supported by China STEM Journal Excellence Action Plan (C-099).

\section{Declarations}

Conflict of interest YLL, XSY, and YZY are medical editors of the journal. ZZY and SQ are Chief Editors of the journal. All authors declare that they have no financial or non-financial conflict of interest related to this paper.

Ethical approval Not needed.

\section{References}

1. Zhang ZH. Nation moves to reinforce research integrity. http://www.chinadaily.com.cn/a/202001/10/WS5e17ccd0a310cf3e355839fb.html Accessed 5 Nov 2021.

2. Guidelines on promoting scientific integrity. http://www.gov.cn/ zhengce/2018-05/30/content_5294886.htm. Accessed 5 Nov 2021.

3. Tang L. Five ways China must cultivate research integrity. Nature. 2019;575:589-91.

4. Cases with scientific misconduct. https://www.orichina.cn/chann els/6.html. Accessed 5 Nov 2021. 
5. Yang LL, Xu SY, Shao JF, Wang PZ, Wang XJ, Qi Q, et al. Declaration of conflict of interest in medical researchers: a cross-sectional study from China. Learn Publ. 2021. https://doi.org/10.1002/leap.1419.

6. Als-Nielsen B, Chen W, Gluud C, Kjaergard LL. Association of funding and conclusions in randomized drug trials: a reflection of treatment effect or adverse events? JAMA. 2003;290:921-8.

7. Yang L, Wang P, Yang R. Conflict of interest reporting in biomedical journals published in China. Account Res. 2017;24:451-7.

8. Wislar JS, Flanagin A, Fontanarosa PB, Deangelis CD. Honorary and ghost authorship in high impact biomedical journals: a cross sectional survey. BMJ. 2011;343:d6128.

9. CRediT author statement. https://www.elsevier.com/authors/ policies-and-guidelines/credit-author-statement. Accessed 5 Nov 2021.
10. Edlavitch SA. Publishing negative findings and the challenge of avoiding type II errors in studies of suspect teratogens: example of a recent ondansetron publication. Reprod Toxicol. 2017;67:125-8.

11. Kretser A, Murphy D, Bertuzzi S, Abraham T, Allison DB, Boor $\mathrm{KJ}$, et al. Scientific integrity principles and best practices: recommendations from a scientific integrity consortium. Sci Eng Ethics. 2019;25:327-55.

12. Corrections and retractions. https://www.springer.com/gp/edito rial-policies/corrections-and-retractions. Accessed 5 Nov 2021.

Publisher's Note Springer Nature remains neutral with regard to jurisdictional claims in published maps and institutional affiliations. 